\title{
Article \\ Stochastic Behavior of a Two-Unit Parallel System with Dissimilar Units and Op- tional Vacations under Poisson Shocks
}

\author{
Mohamed S. El-Sherbeny ${ }^{1,2, *}$ and Zienab M. Hussien ${ }^{1}$
}

1 Department of Mathematics, Faculty of Sciences and Arts-Rabigh Campus, King Abdulaziz University, Jeddah, Saudi Arabia; $\underline{m}$ el sherbeny@yahoo.com, z.m.hussien@gmail.com.

2 Department of Mathematics, Faculty of Science, Helwan University, Cairo, Egypt.

* Correspondence: $\underline{m}$ el sherbeny@yahoo.com; Tel.: (00966597142527)

\begin{abstract}
This article examines the impact of some system parameters on an industrial system composed of two dissimilar parallel units with one repairman. The active unit may fail due to essential factors like aging or deteriorating, or exterior phenomena such as Poisson shocks that occur at various time periods. Whenever the value of a shock is larger than the specified threshold of the active unit, the active unit will fail. The article assumes that the repairman has the right to take any of two decisions at the beginning of the system operation: either a takes a vacation if the two units work in a normal way, or stay in the system to monitor the system until the first system failure. In case of having a failure in any of the two units during the absence of the repairman, the failing unit will have to wait until the repairman is called back to work. We suppose that the value of every shock is assumed to be i.i.d. with some known distribution. The length of the repairman's vacation, repair time, and recall time are arbitrary distributions. Various reliability measures have been calculated by the supplementary variable technique and the Markov's vector process theory. At last, numerical computation and graphical analysis have been given for a particular case to validate the derived indices.
\end{abstract}

Keywords: Mean time to failure; Poisson shock; Steady-state availability; Steady-state frequency; Supplementary variable technique.

\section{Introduction}

The economic progress of any country depends to a great extent on developing the industrial and mechanical fields. This progress leads to the appearance of new technicalities that help solving problems that may occur in these complex industrial systems. But in spite of all that help, consumers hope to deal with low cost and high efficiency industrial systems. Therefore, system designers and researchers in this field face a great challenge to develop many systems and raise their efficiency, reliability, and safety.

Many researches have been made in the past to explain the concept of reliability and to raise the efficiency of many industrial systems, and to analyze the cost of the different redundant systems under the effect of some restrictions such as the periods of which the system is on or off, the different types of failures affecting that system, the different types of repairing these units, whether or not it's better for the repair man to take single or multiple vacations...etc.

In this context, we present some of the previous research work that deserves mention. The concept of vacation was first presented to the model analysis of the queuing system in article [1] as 2-standard vacation policies. These policies were defined as multiple vacations and single vacation. Some thorough and excellent studies from the modern results for a variety of vacation models, inclusive of some applications were presented by [2]. Many researchers, though, only studied different vacation models from a queuing theory viewpoint. Therefore, in article [3] the concept of vacation was introduced by the reliability theory viewpoint, and an n-component series system with multiple vacations of repairman was discussed. Since then, the researchers were interested in 
studying reliability models with the vacation. The reader can see e.g. $[4,5,6,7,8,9]$. The 2-component repairable systems are one of the meaningful reliability applications. Therefore, many authors have studied various repairable systems and obtained many measures of reliability in those systems. For example, [10] used the Markov renewal theory to study the behavior of the system which consists of a 2- parallel component in the presence of the maintenance man. [11] applied the reliability theory on a two-component series repairable system. The system can be used to analyze the reliability measures of some automatic systems. The problem of repairing industrial equipment with two vacation policies (single vacation and multiple vacations) is studied in article [12] using queuing theory.

Most contemporary researchers focused on repairable systems under the effect of Poisson shocks. For example, [13] discussed the effect of Poisson Shocks on the behavior of a repairable system which consists of two units, one of them is working and the other is cold standby with a single repairman. [14] analyzed the reliability of the repairable system under the influence of two types of failure. One of them is external factors such as Poisson shocks and the other is fundamental factors such as aging or deterioration. The repairman has the right to take a vacation when the system is active. [15] displayed a two non-identical unit cold standby repairable system maintained by a single repairman. The proposed system suffers from one of two types of failures. One has to do with intrinsic factors, and the other is related to external shocks such as the stepwise Poisson process. [16] focuses on one unit under Poisson shocks with the assumption that the deterioration caused by a single shock may be ineffective, and the system fails only when the deterioration has accumulated to a specific level. The effect Poisson shocks has on the system behavior, which consists of two-non-symmetric parallel units, one of them is operative and the other is cold standby, with the presence of the imperfect key switch between these two units, was investigated by [17].

The reliability measurements of two dissimilar parallel units for the repairable systems are important to the industry field. Analyzing and elicitation of these measurements for the systems influenced by shocks can be more motivating and interesting as chocks take place from time to time in the world. This induces us to derive the reliability of two dissimilar parallel units for a repairable system. In this article, we assume the system might be subjected to shocks following a Poisson process and the repairman has the choice to either stay at the system to monitor the two units until one fails or takes a vacation if the two units work in a normal way. It should be noted that system behavior analysis is not an easy task when all system distributions are general distributions.

In this article, we present the supplementary variable method that helps in solving partial differential equations related to describe the dynamics of movement between the different states of the system. With the help of the ergodicity of the investigated process and supplementary variable method, we obtain explicit expressions of reliability metrics such as reliability function, steady-state availabilities, mean time to system failure, Steady-state probability that the repairman is on vacation, Steady-state probability that the system is waiting for repair, and Steady-state failure frequency.

This article is designed as follows:

Section 2 introduces more details about the system and assumptions of the system description. Section 3 deduces integro-differential equations which describe the movement between the different states of the system. The reliability measures for this system are calculated in section 4 . Section 5 introduces a special case; the repairman stays in the system without taking any vacation. The numerical examples are presented in section 6 . Conclusive remarks are offered in the final section. 


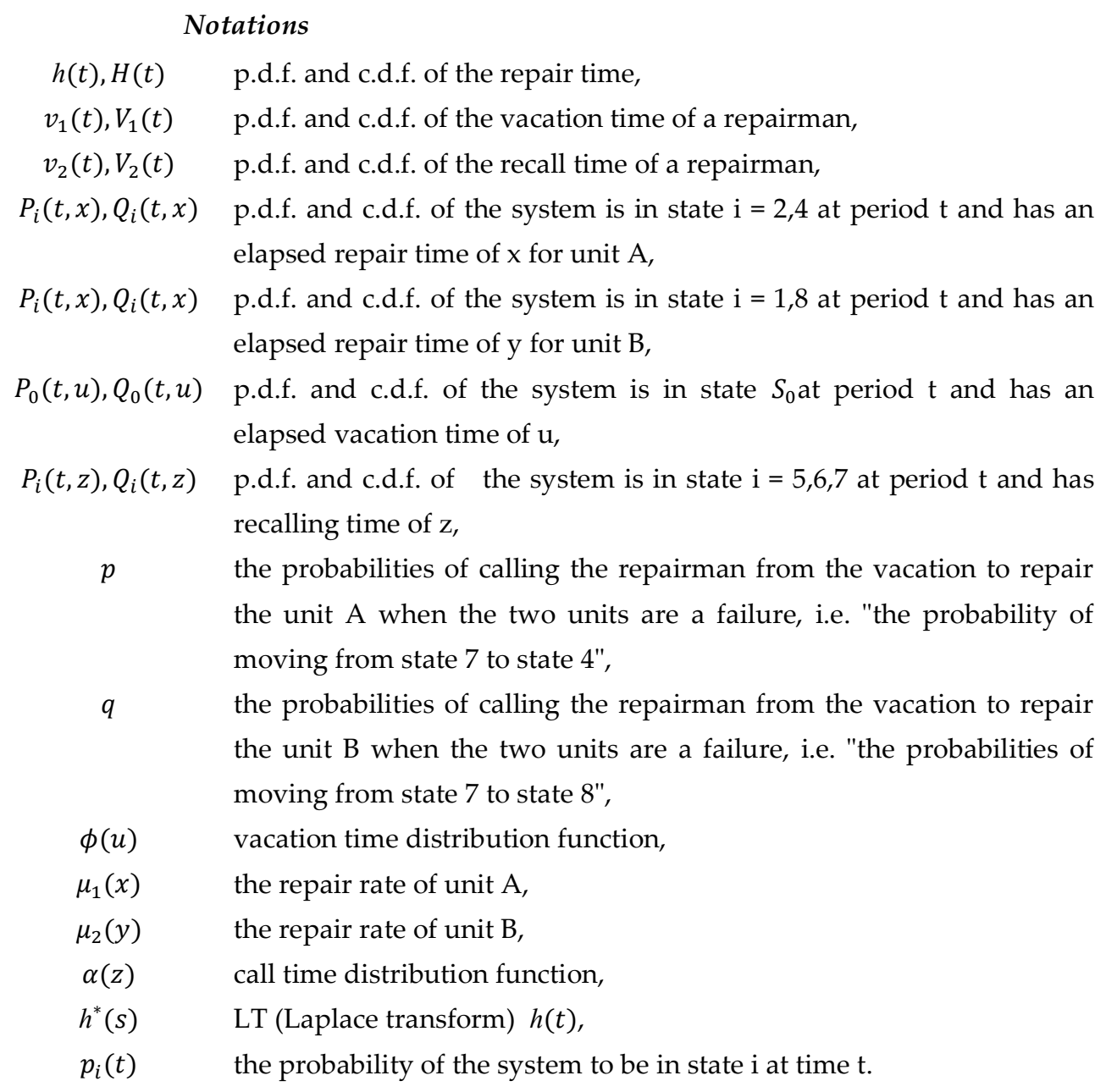

\section{Description of the System and Assumptions}

The system which consists of two dissimilar units and a single repairman under Poisson shock is subjected to the following assumptions:

A1: At the initial time, both units are working with high efficiency, and a repairman has the choice to either stay in the system or takes a vacation.

A2: The system is exposed to shocks continually. The arrival of shocks is considered as a Poisson process $\{S(t), t \geq 0\}$ with the strength $\lambda_{i}>0$. The value of every shock is $\hat{Y}$, i.i.d random variable with distribution function $F$.

A3: When a shock occurs and the value of this shock overrides a threshold, the active unit will break down. The threshold of units (A and B) is a non-negative random variable $\tau_{i}$ with a distribution function $\xi$.

A4: When any unit fails with the existence of the repairman in the system, it will be repaired immediately. Once the repairman is done repairing the failed units, the repairman has the choice to either stay at the system or take a vacation, then he returns from vacation if at least one unit is failing. The repair rule is "first-in-first-out". If a unit fails while the other is being repaired, the recently failed unit must wait for repair, and the system has to stop working. To expedite the system operation when the repairman in a vacation and the system is a breakdown, the system requires repairing one of two units A or B, with probability p or $q$ respectively.

A5: Shocks are the main reason for units to fail, and the system fails only if both the units fail. 
Based on the preceding assumptions, we can conclude that the conditional failure probability of unit $\mathrm{A}$ and unit $\mathrm{B}$ are random variables $\xi_{i}(\hat{Y})(i=1,2)$.

The probability distributions are:

$$
K_{i}(y)=P\left(\xi_{i}(\hat{Y}) \leq y\right)=P\left(\hat{Y} \leq \xi_{i}^{-1}(y)\right)=F\left(\xi_{i}^{-1}(y)\right), 0 \leq y \leq 1 .(i=1,2) .
$$

The possibility of a single shock causes unit (A or B) to fail. According to the above assumptions $A_{2}, A_{3}$, we get

$$
\tau_{i}=P\left(\hat{Y} \leq \tau_{i}\right)=\int_{0}^{\infty} P\left(\tau_{i}<\hat{y} \mid \hat{Y}=\hat{y}\right) d P(\hat{Y} \leq \hat{y})=\int_{0}^{\infty} \xi_{i}(\hat{y}) d F(\hat{y}), 0 \leq y \leq 1 .(i=1,2) .
$$

\section{System Analysis}

The states of this system $\Omega(t)$ at time $t$ as a following:

$S_{0}$ : at any time $t$, unit $A$ is active, unit $B$ is active, and the repairman has the choice to either stay at the system or takes a vacation.

$S_{1}$ : at any time $t$, unit $A$ is active unit $B$ is being repaired, and the repairman still in the system, the system is working.

$S_{2}$ : at any time $t$ unit $A$ is being repaired, unit $B$ is active, and the repairman still in the system, the system is working.

$S_{3}$ : at any time $t$, unit $A$ is active, unit $B$ is active, and the repairman chooses to take a vacation, the system is working.

$S_{4}$ : at any time $t$, unit $A$ is still repaired from above $S 2$, unit B is waiting for being repaired, and the system is down.

$S_{5}$ : at any time $t$, unit A is active, unit B is waiting for repair, the repairman in a vacation, and the system is working.

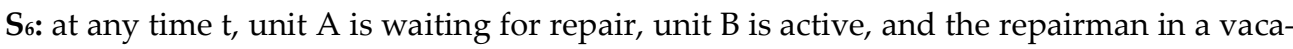
tion, the system is working.

$S_{7}$ : at any time $t$, unit A is still waiting for repair from S6, unit B is waiting for repair and the repairman in a vacation either go to state 4 with probability p or going to state 8 with probability $\mathrm{q}$ and the system is down.

Ss: at any time $t$, unit $A$ is waiting for being repaired, unit $B$ is under repair and the system is down.

The state space is $E=\left\{S_{0}, S_{1}, S_{2}, S_{3}, S_{4}, S_{5}, S_{6}, S_{7}, S_{8}\right\}$, where the working state is $U=$ $\left\{S_{0}, S_{1}, S_{2}, S_{3}, S_{5}, S_{6}\right\}$ and the down state is $D=\left\{S_{4}, S_{7}, S_{8}\right\}$. Accordance with the above assumptions, and $\{\Omega(t), t \geq 0\}$ is not a Markov process we using the supplementary variables as a following:

$X_{1}(t):$ is time to make a vacation decision. $\Omega(t)=\left\{S_{0}\right\}$.

$X_{2}(t)$ : is the elapsed vacation time. $\Omega(t)=\left\{S_{5}, S_{6}, S_{7}\right\}$.

$Y_{1}(t)$ : is the elapsed repair time of unit A being repaired at time t. $\Omega(t)=\left\{S_{2}, S_{4}\right\}$.

$Y_{2}(t)$ : is the elapsed repair time of unit B being repaired at time t. $\Omega(t)=\left\{S_{1}, S_{8}\right\}$.

State space is a following: $\Omega^{*}=\{(0, u),(1, y),(2, x), 3,(4, x),(5, z),(6, z),(7, z),(8, y)\}$ where $u, z, x$ and yare explanatory values of $X_{1}(t), X_{2}(t), Y_{1}(t)$ and $Y_{2}(t)$, respectively. Indicate to:

$Q_{0}(t, u)=\rho\left(\Omega(t)=0, X_{1}(t) \leq u\right), Q_{i}(t, z)=\rho\left(\Omega(t)=i, X_{2}(t) \leq z\right),(i=5,6,7)$,

$Q_{i}(t, x)=\rho\left(\Omega(t)=i, Y_{1}(t) \leq x\right),(i=2,4), Q_{i}(t, y)=\rho\left(\Omega(t)=i, Y_{2}(t) \leq y\right),(i=1,8)$.

where $\rho(E)$ is probability of event $E$. 
$\rho_{i}(t, w)=\frac{d}{d w} Q_{i}(t, w),(i=0,1,2,4,5,6,7,8)$.

$\varphi_{i}(t)=\rho(s(t)=i),(i=0,1,2,4,5,6,7,8)$.

From the above, we can formulation the differential equations that represent this system by using the probability arguments and limiting transitions as following.

$$
\rho_{0}(t+\Delta t, u+\Delta t)=\rho_{0}(t, u)\left(1-\left(\lambda_{1} r_{1}+\lambda_{2} r_{2}+\phi(u)\right) \Delta t\right)+o \Delta t
$$

When $\Delta t$ tend to zero, we get

$\left(\frac{\partial}{\partial t}+\frac{\partial}{\partial u}+\lambda_{1} r_{1}+\lambda_{2} r_{2}+\phi(u)\right) \rho_{0}(t, u)=0$,

The same style, we get the following:

$$
\begin{aligned}
& \left(\frac{\partial}{\partial t}+\frac{\partial}{\partial y}+\lambda_{1} r_{1}+\mu_{2}(y)\right) \rho_{1}(t, y)=0 \\
& \left(\frac{\partial}{\partial t}+\frac{\partial}{\partial x}+\lambda_{2} r_{2}+\mu_{1}(x)\right) \rho_{2}(t, x)=0 \\
& \left(\frac{d}{d t}+\lambda_{1} r_{1}+\lambda_{2} r_{2}\right) \varphi_{3}(t)=\int_{0}^{\infty} \rho_{0}(u, t) \phi(u) d u \\
& \left(\frac{\partial}{\partial t}+\frac{\partial}{\partial x}+\mu_{1}(x)\right) \rho_{4}(t, x)=\lambda_{2} r_{2} \rho_{2}(t, x) \\
& \left(\frac{\partial}{\partial t}+\frac{\partial}{\partial z}+\lambda_{1} r_{1}+\alpha(z)\right) \rho_{5}(t, z)=0 \\
& \left(\frac{\partial}{\partial t}+\frac{\partial}{\partial z}+\lambda_{2} r_{2}+\alpha(z)\right) \rho_{6}(t, z)=0 \\
& \left(\frac{\partial}{\partial t}+\frac{\partial}{\partial z}+\alpha(z)\right) \rho_{7}(t, z)=\lambda_{1} r_{1} \rho_{5}(t, z)+\lambda_{2} r_{2} \rho_{6}(t, z) \\
& \left(\frac{\partial}{\partial t}+\frac{\partial}{\partial y}+\mu_{2}(y)\right) \rho_{8}(t, y)=\lambda_{1} r_{1} \rho_{1}(t, y)
\end{aligned}
$$

The boundary conditions are:

$$
\begin{aligned}
& \rho_{0}(t, 0)=\int_{0}^{\infty} \rho_{1}(t, y) \mu_{2}(y) d y+\int_{0}^{\infty} \rho_{2}(t, x) \mu_{1}(x) d x+\varepsilon(t), \\
& \rho_{1}(t, 0)=\int_{0}^{\infty} \lambda_{2} r_{2} \rho_{0}(t, u) d u+\int_{0}^{\infty} \rho_{4}(t, x) \mu_{1}(x) d x+\int_{0}^{\infty} \rho_{5}(t, z) \alpha(z) d z, \\
& \rho_{2}(t, 0)=\int_{0}^{\infty} \lambda_{1} r_{1} \rho_{0}(t, u) d u+\int_{0}^{\infty} \rho_{8}(t, y) \mu_{2}(y) d y+\int_{0}^{\infty} \rho_{6}(t, z) \alpha(z) d z, \\
& \rho_{5}(t, 0)=\lambda_{2} r_{2} \varphi_{3}(t) \\
& \rho_{6}(t, 0)=\lambda_{1} r_{1} \varphi_{3}(t) \\
& \rho_{7}(t, 0)=0 \\
& \rho_{4}(t, 0)=\rho_{8}(t, 0)=\int_{0}^{\infty} \rho_{7}(t, z) \alpha(z) d z, \\
& \sum_{i=0}^{2} \int_{0}^{\infty} \rho_{i}(t, m) d m+\varphi_{3}(t)+\sum_{i=4}^{8} \int_{0}^{\infty} \rho_{i}(t, m) d m=1, m=u, x, y, z
\end{aligned}
$$

(17)

The initial conditions are:

$$
\rho_{0}(0, u)=\varepsilon(u)=\left\{\begin{array}{ll}
1 & u=0 \\
0 & u \neq 0
\end{array},\right.
$$

$\rho_{0}(0, m)=0, \quad m \neq 0, m=u, x, y, z . i=1, \ldots, 8 . \varphi_{3}(0)=0$.

Laplace transform can be defined as a following:

$$
k^{*}(s)=L\{k(x)\}=\int_{0}^{\infty} k(x) e^{-s x} d x, s>0 .
$$


The ergodicity of the investigation process guarantees the presence of the following steady-probability: $\varphi_{i}=\lim _{t \rightarrow \infty} \quad \varphi_{i}(t), i=1, \ldots, 8 . \theta_{i}(m)=\lim _{t \rightarrow \infty} \rho_{i}(t, m), i=$ $1,2,4,5,6,7,8$. which follows the following relations: $\varphi_{i}=\int_{0}^{\infty} \theta_{i}(m) d m, \quad i=$ $1,2,4,5,6,7,8$.

Taking the limit $t \rightarrow \infty$ in the equations (1) - (17), the following equations are obtained:

$\left(\frac{d}{d u}+\lambda_{1} r_{1}+\lambda_{2} r_{2}+\phi(u)\right) \theta_{0}(u)=0$

$\left(\frac{d}{d y}+\lambda_{1} r_{1}+\mu_{2}(y)\right) \theta_{1}(y)=0$

$\left(\frac{\partial}{\partial x}+\lambda_{2} r_{2}+\mu_{1}(x)\right) \theta_{2}(x)=0$,

$\left(\lambda_{1} r_{1}+\lambda_{2} r_{2}\right) \varphi_{3}=\int_{0}^{\infty} \theta_{0}(u) \phi(u) d u$,

$\left(\frac{d}{d x}+\mu_{1}(x)\right) \theta_{4}(x)=\lambda_{2} r_{2} \theta_{2}(x)$,

$\left(\frac{d}{d z}+\lambda_{1} r_{1}+\alpha(z)\right) \theta_{5}(z)=0$

$\left(\frac{\partial}{\partial z}+\lambda_{2} r_{2}+\alpha(z)\right) \theta_{6}(z)=0$

$\left(\frac{d}{d z}+\alpha(z)\right) \theta_{7}(z)=\lambda_{1} r_{1} \theta_{5}(z)+\lambda_{2} r_{2} \theta_{6}(z)$

$\left(\frac{d}{d y}+\mu_{2}(y)\right) \theta_{8}(y)=\lambda_{1} r_{1} \theta_{1}(y)$

$\theta_{0}(0)=\int_{0}^{\infty} \theta_{1}(y) \mu_{2}(y) d y+\int_{0}^{\infty} \theta_{2}(x) \mu_{1}(x) d x$,

$\theta_{1}(0)=\int_{0}^{\infty} \lambda_{2} r_{2} \theta_{0}(u) d u+\int_{0}^{\infty} \theta_{4}(x) \mu_{1}(x) d x+\int_{0}^{\infty} \theta_{5}(z) \alpha(z) d z$,

$\theta_{2}(0)=\int_{0}^{\infty} \lambda_{1} r_{1} \theta_{0}(u) d u+\int_{0}^{\infty} \theta_{8}(y) \mu_{2}(y) d y+\int_{0}^{\infty} \theta_{6}(z) \alpha(z) d z$,

$\theta_{5}(0)=\lambda_{2} r_{2} \varphi_{3}$

$\theta_{6}(0)=\lambda_{1} r_{1} \varphi_{3}$

$\theta_{7}(0)=0$,

$\theta_{4}(0)=\theta_{8}(0)=\int_{0}^{\infty} \theta_{7}(z) \alpha(z) d z$,

$\sum_{i=0}^{2} \int_{0}^{\infty} \theta_{i}(m) d m+\varphi_{3}+\sum_{i=4}^{8} \int_{0}^{\infty} \theta_{i}(m) d m=1, m=u, x, y, z$.

(34)

\section{Reliability Characteristics}

According to the results derived from the analysis of the system in the previous section, the reliability index of the system is obtained as follows:

4.1. Steady-state availability is

$A v(\infty)=\sum_{i=0}^{3} \varphi_{i}+\sum_{i=5}^{6} \varphi_{i}$

(35)

4.2. Steady-state probability that the repairman is on vacation is

$\varphi_{\text {rep.V }}=\varphi_{3}+\sum_{i=5}^{7} \varphi_{i}$

(36)

4.3. Steady-state probability that the system is waiting for repair is $\varphi_{\text {rep. } W}=\varphi_{7}$. 
4.4. Steady-state failure frequency is

$f . f=\lambda_{1} r_{1}\left(\varphi_{1}+\varphi_{5}\right)+\lambda_{2} r_{2}\left(\varphi_{2}+\varphi_{6}\right)$.

(38)

where,

The steady-state probabilities can be obtained as follows from $\varphi_{i}=\int_{0}^{\infty} \theta_{i}(m) d m$, for all $i=1,2,4,5,6,7,8$.

$\varphi_{0}=C_{0} \bar{V}_{1}^{*}\left(\lambda_{1} r_{1}+\lambda_{2} r_{2}\right)$,

$\varphi_{1}=\left\{C_{0} \bar{H}_{2}^{*}\left(r_{1} \lambda_{1}\right)\left(v_{1}^{*}\left(\lambda_{1} r_{1}+\lambda_{2} r_{2}\right)\left(\lambda_{2} r_{2}\left(p+q+q h_{1}^{*}\left(\lambda_{2} r_{2}\right)\left(v_{2}^{*}\left(\lambda_{1} r_{1}\right)-1\right)-(p+q-\right.\right.\right.\right.$

1) $\left.v_{2}^{*}\left(\lambda_{1} r_{1}\right)\right)$

$\left.+\lambda_{1} r_{1}\left(p+q-(p+q-1) v_{2}^{*}\left(\lambda_{2} r_{2}\right)+h_{1}^{*}\left(\lambda_{2} r_{2}\right)\left((q-1) v_{2}^{*}\left(\lambda_{2} r_{2}\right)-q\right)\right)\right)-\left(\lambda_{1} r_{1}+\lambda_{2} r_{2}\right)\left(\lambda_{1} r_{1}-\lambda_{2} r_{2}\right.$

$\frac{\left.\left.\left.\left(h_{1}^{*}\left(\lambda_{2} r_{2}\right)-1\right)\right) \bar{V}_{1}^{*}\left(\lambda_{1} r_{1}+\lambda_{2} r_{2}\right)\right)\right)}{\left(\left(\lambda_{1} r_{1}+\lambda_{2} r_{2}\right)\left(h_{2}^{*}\left(\lambda_{1} r_{1}\right)-h_{1}^{*}\left(\lambda_{2} r_{2}\right)\left(h_{2}^{*}\left(\lambda_{1} r_{1}\right)-1\right)\right)\right)^{\prime}}$

$\phi_{2}=-\left\{C_{0} \bar{H}_{1}^{*}\left(r_{2} \lambda_{2}\right)\left(v_{1}^{*}\left(\lambda_{1} r_{1}+\lambda_{2} r_{2}\right)\left(\lambda_{2} r_{2}\left(p+q-(p+q-1) v_{2}^{*}\left(\lambda_{1} r_{1}\right)+h_{2}^{*}\left(\lambda_{1} r_{1}\right)\left((p-1) v_{2}^{*}\left(\lambda_{1} r_{1}\right)-p\right)\right)\right.\right.\right.$

$\left.+\lambda_{1} r_{1}\left(p+q+p h_{2}^{*}\left(\lambda_{1} r_{1}\right)\left(v_{2}^{*}\left(\lambda_{2} r_{2}\right)-1\right)-(p+q-1) v_{2}^{*}\left(\lambda_{2} r_{2}\right)\right)\right)+\left(\lambda_{1} r_{1}+\lambda_{2} r_{2}\right)\left(\lambda_{1} r_{1}-\lambda_{2} r_{2}\right.$

$\frac{\left.\left.\left.\left(h_{1}^{*}\left(\lambda_{1} r_{1}\right)-1\right)\right) \bar{V}_{1}^{*}\left(\lambda_{1} r_{1}+\lambda_{2} r_{2}\right)\right)\right)}{\left(\left(\lambda_{1} r_{1}+\lambda_{2} r_{2}\right)\left(h_{2}^{*}\left(\lambda_{2} r_{2}\right)\left(h_{2}^{*}\left(\lambda_{1} r_{1}\right)-1\right)-h_{2}^{*}\left(\lambda_{1} r_{1}\right)\right)\right)^{\prime}}$

$\varphi_{3}=\frac{C_{0} v_{1}^{*}\left(\lambda_{1} r_{1}+\lambda_{2} r_{2}\right)}{\left(\lambda_{1} r_{1}+\lambda_{2} r_{2}\right)}$

$\varphi_{4}=\frac{C_{0}\left(-p v_{1}^{*}\left(\lambda_{1} r_{1}+\lambda_{2} r_{2}\right)\left(\lambda_{2} r_{2}\left(v_{2}^{*}\left(\lambda_{1} r_{1}\right)-1\right)+\lambda_{1} r_{1}\left(v_{2}^{*}\left(\lambda_{2} r_{2}\right)-1\right)\right)\right.}{\left(\lambda_{1} r_{1}+\lambda_{2} r_{2}\right) \mu_{1}}+\frac{1}{h_{1}^{*}\left(\lambda_{2} r_{2}\right)\left(h_{2}^{*}\left(\lambda_{1} r_{1}\right)-1\right)-h_{2}^{*}\left(\lambda_{1} r_{1}\right)}$

$\left(\mu_{1} \bar{H}_{1}^{*}\left(\lambda_{2} r_{2}\right)-1\right)\left(v_{1}^{*}\left(\lambda_{1} r_{1}+\lambda_{2} r_{2}\right)\left(\lambda_{2} r_{2}\left(p+q-(p+q-1) v_{2}^{*}\left(\lambda_{1} r_{1}\right)-h_{2}^{*}\left(\lambda_{1} r_{1}\right)\left(q v_{2}^{*}\left(\lambda_{1} r_{1}\right)+p\right)\right)\right.\right.$

$\left.+\lambda_{1} r_{1}\left(p+q+p h_{2}^{*}\left(\lambda_{1} r_{1}\right)\left(v_{2}^{*}\left(\lambda_{2} r_{2}\right)-1\right)-(p+q-1) v_{2}^{*}\left(\lambda_{2} r_{2}\right)\right)\right)+\left(\lambda_{1} r_{1}+\lambda_{2} r_{2}\right)\left(\lambda_{1} r_{1}-\lambda_{2} r_{2}\right.$

$\left.\left.\left.\left(h_{2}^{*}\left(\lambda_{1} r_{1}\right)-1\right)\right) \bar{V}_{1}^{*}\left(\lambda_{1} r_{1}+\lambda_{2} r_{2}\right)\right)\right)$

$\varphi_{5}=\frac{C_{0} \lambda_{2} r_{2} v_{1}^{*}\left(\lambda_{1} r_{1}+\lambda_{2} r_{2}\right) \bar{V}_{2}^{*}\left(\lambda_{1} r_{1}\right)}{\left(\lambda_{1} r_{1}+\lambda_{2} r_{2}\right)}$

$\varphi_{6}=\frac{C_{0} \lambda_{1} r_{1} v_{1}^{*}\left(\lambda_{1} r_{1}+\lambda_{2} r_{2}\right) \bar{V}_{2}^{*}\left(\lambda_{2} r_{2}\right)}{\left(\lambda_{1} r_{1}+\lambda_{2} r_{2}\right)}$

$\varphi_{7}=\frac{C_{0} v_{1}^{*}\left(\lambda_{1} r_{1}+\lambda_{2} r_{2}\right)\left(\lambda_{2} r_{2}\left(1-\alpha \bar{V}_{2}^{*}\left(\lambda_{1} r_{1}\right)\right)+\lambda_{1} r_{1}\left(1-\alpha \bar{V}_{2}^{*}\left(\lambda_{2} r_{2}\right)\right)\right)}{\alpha\left(\lambda_{1} r_{1}+\lambda_{2} r_{2}\right)}$,

$\varphi_{8}=\frac{C_{0}}{\left(\lambda_{1} r_{1}+\lambda_{2} r_{2}\right)}\left\{\frac{-q v_{1}^{*}\left(\lambda_{1} r_{1}+\lambda_{2} r_{2}\right)\left(\lambda_{2} r_{2}\left(v_{1}^{*}\left(\lambda_{1} r_{1}\right)-1\right)+\lambda_{1} r_{1}\left(v_{2}^{*}\left(\lambda_{2} r_{2}\right)-1\right)\right)}{\mu_{2}}+\right.$

$\frac{\left(\frac{1}{\mu_{2}}-\bar{H}_{2}^{*}\left(\lambda_{1} r_{1}\right)\right)}{h_{1}^{*}\left(\lambda_{2} r_{2}\right)\left(1-h_{2}^{*}\left(\lambda_{1} r_{1}\right)\right)+h_{2}^{*}\left(\lambda_{1} r_{1}\right)}\left(v_{1}^{*}\left(\lambda_{1} r_{1}+\lambda_{2} r_{2}\right)\left(\lambda_{2} r_{2}\left(p+q+q h_{1}^{*}\left(\lambda_{2} r_{2}\right)\left(\left(v_{2}^{*}\left(\lambda_{1} r_{1}\right)-1\right)\right)\right.\right.\right.$ 


$$
\begin{aligned}
& \left.-(p+q-1) v_{2}^{*}\left(\lambda_{1} r_{1}\right)\right) \\
& \quad+\lambda_{1} r_{1}\left(p+q-(p+q-1) v_{2}^{*}\left(\lambda_{2} r_{2}\right)+h_{1}^{*}\left(\lambda_{2} r_{2}\right)\left(-q+(q-1) v_{2}^{*}\left(\lambda_{2} r_{2}\right)\right)\right) \\
& \left.-\left(\lambda_{1} r_{1}+\lambda_{2} r_{2}\right)\left(-\lambda_{2} r_{2}+\lambda_{1} r_{1}\left(h_{1}^{*}\left(\lambda_{2} r_{2}\right)-1\right)\right) \bar{V}_{1}^{*}\left(\lambda_{1} r_{1}+\lambda_{2} r_{2}\right)\right) \\
& C_{0}=\left\{\left(\lambda_{1} r_{1}+\lambda_{2} r_{2}\right) \mu_{1} \mu_{2}\left(h_{1}^{*}\left(\lambda_{2} r_{2}\right)\left(h_{2}^{*}\left(\lambda_{1} r_{1}\right)-1\right)-h_{2}^{*}\left(\lambda_{1} r_{1}\right)\right)\right\} /\left\{\frac { 1 } { \alpha } v _ { 1 } ^ { * } ( \lambda _ { 1 } r _ { 1 } + \lambda _ { 2 } r _ { 2 } ) \left(\alpha \mu_{1} \mu_{2} h_{1}^{*}\left(\lambda_{2} r_{2}\right)\right.\right. \\
& \left.\left(h_{2}^{*}\left(\lambda_{1} r_{1}\right)-1\right)-h_{2}^{*}\left(\lambda_{1} r_{1}\right)\right)+\lambda_{2} r_{2}\left(-(p+q) \alpha\left(\mu_{1}+\mu_{2}\right)-\left(p \alpha+\mu_{1}\right) \mu_{2} h_{1}^{*}\left(\lambda_{2} r_{2}\right)+\left(\mu_{1}(q \alpha+\right.\right. \\
& \left.\left.\left.\left.\mu_{2}\right) \quad \mu_{1}\left(-1+p+q+h_{1}^{*}\left(\lambda_{2} r_{2}\right)-q\left(h_{1}^{*}\left(\lambda_{2} r_{2}\right)-1\right) h_{2}^{*}\left(\lambda_{1} r_{1}\right)\right)\right) v_{2}^{*}\left(\lambda_{2} r_{2}\right)\right)\right) \\
& \left.\quad\left(h_{1}^{*}\left(\lambda_{2} r_{2}\right)-1\right)+p \alpha \mu_{2} h_{1}^{*}\left(\lambda_{2} r_{2}\right)\right) h_{2}^{*}\left(\lambda_{1} r_{1}\right)+\alpha\left(\mu_{2}\left(-1+p+q-p h_{1}^{*}\left(\lambda_{2} r_{2}\right)\left(h_{2}^{*}\left(\lambda_{1} r_{1}\right)-1\right)\right)+\right. \\
& \quad+\left(\lambda_{1} r_{1}+\lambda_{2} r_{2}\right)\left(\lambda _ { 1 } r _ { 1 } \left(-\mu_{2}+\right.\right. \\
& \left.\mu_{1}\left(-1+h_{1}^{*}\left(\lambda_{2} r_{2}\right)\right)\right)-\lambda_{2} r_{2}\left(\mu_{1}+\mu_{2}-\mu_{2} h_{2}^{*}\left(\lambda_{1} r_{1}\right)\right)+\mu_{1} \mu_{2}\left(-h_{1}^{*}\left(\lambda_{2} r_{2}\right)+\left(h_{1}^{*}\left(\lambda_{2} r_{2}\right)-1\right) h_{2}^{*}\left(\lambda_{2} r_{2}\right)\right) \\
& \left.V_{1}^{*}\left(\lambda_{1} r_{1}+\lambda_{2} r_{2}\right)\right\} .
\end{aligned}
$$

\section{Mean Time to the First Failure (MTTFF)}

In this section, we deduce the mean time to the first failure (MTTFF) of the system. We assumed that $\mathrm{t}$ be the time to the first failure of the system, therefore the reliability function of this system is calculated as follows $R(t)=P(\tau>t)$. To obtain the reliability function, we consider the failure states $\{4,7,8\}$ of the system are absorbing states.

Let:

$$
\begin{aligned}
& L_{0}(t, u)=\frac{d}{d u} \rho\left[\tilde{S}(t)=0, \tilde{X}_{1}(t) \leq u\right], \quad L_{i}(t, u)=\frac{d}{d z} \rho\left[\tilde{S}(t)=i, \tilde{X}_{2}(t) \leq z\right], i=5,6 . \\
& L_{2}(t, x)=\frac{d}{d x} \rho\left[\tilde{S}(t)=2, \tilde{Y}_{1}(t) \leq x\right], \quad L_{1}(t, y)=\frac{d}{d y} \rho\left[\tilde{S}(t)=1, \tilde{Y}_{2}(t) \leq y\right], \quad L_{3}(t)= \\
& \rho[\tilde{S}(t)=3] .
\end{aligned}
$$

In the same manner as previously mentioned in Section 4, we conclude reliability function as following:

$$
\begin{aligned}
& \left(\frac{\partial}{\partial t}+\frac{\partial}{\partial u}+\lambda_{1} r_{1}+\lambda_{2} r_{2}+\phi(u)\right) L_{0}(t, u)=0 \\
& \left(\frac{\partial}{\partial t}+\frac{\partial}{\partial y}+\lambda_{1} r_{1}+\mu_{2}(y)\right) L_{1}(t, y)=0 \\
& \left(\frac{\partial}{\partial t}+\frac{\partial}{\partial x}+\lambda_{2} r_{2}+\mu_{1}(x)\right) L_{2}(t, x)=0 \\
& \left(\frac{d}{d t}+\lambda_{1} r_{1}+\lambda_{2} r_{2}\right) L_{3}(t)=\int_{0}^{\infty} L_{0}(u, t) \phi(u) d u \\
& \left(\frac{\partial}{\partial t}+\frac{\partial}{\partial z}+\lambda_{1} r_{1}+\alpha(z)\right) L_{5}(t, z)=0 \\
& \left(\frac{\partial}{\partial t}+\frac{\partial}{\partial z}+\lambda_{2} r_{2}+\alpha(z)\right) L_{6}(t, z)=0
\end{aligned}
$$

The boundary conditions are: 
$L_{0}(t, 0)=\int_{0}^{\infty} L_{1}(t, y) \mu_{2}(y) d y+\int_{0}^{\infty} L_{2}(t, x) \mu_{1}(x) d x+\varepsilon(t)$,

$L_{1}(t, 0)=\int_{0}^{\infty} \lambda_{2} r_{2} L_{0}(t, u) d u+\int_{0}^{\infty} L_{5}(t, z) \alpha(z) d z$,

$L_{2}(t, 0)=\int_{0}^{\infty} \lambda_{1} r_{1} L_{0}(t, u) d u+\int_{0}^{\infty} L_{6}(t, z) \alpha(z) d z$,

$L_{5}(t, 0)=\lambda_{2} r_{2} L_{3}(t)$,

$L_{6}(t, 0)=\lambda_{1} r_{1} L_{3}(t)$,

The initial conditions are:

$$
R_{0}(0, u)=\varepsilon(u)=\left\{\begin{array}{ll}
1 & u=0 \\
0 & u \neq 0
\end{array},\right.
$$

Taking the Laplace transform of the equations (39-49), as well as initial conditions, we have:

$$
\begin{aligned}
& \left(\frac{d}{d u}+s+\lambda_{1} r_{1}+\lambda_{2} r_{2}+\phi(u)\right) L_{0}^{*}(s, u)=0, \\
& \left(\frac{d}{d y}+s+\lambda_{1} r_{1}+\mu_{2}(y)\right) L_{1}^{*}(s, y)=0, \\
& \left(\frac{\partial}{\partial x}+s+\lambda_{2} r_{2}+\mu_{1}(x)\right) L_{2}^{*}(s, x)=0, \\
& \left(s+\lambda_{1} r_{1}+\lambda_{2} r_{2}\right) L_{3}^{*}(s)=\int_{0}^{\infty} L_{0}^{*}(u, s) \phi(u) d u, \\
& \left(\frac{d}{d z}+s+\lambda_{1} r_{1}+\alpha(z)\right) L_{5}^{*}(s, z)=0, \\
& \left(\frac{d}{d z}+s+\lambda_{2} r_{2}+\alpha(z)\right) L_{6}^{*}(s, z)=0, \\
& L_{0}^{*}(s, 0)=\int_{0}^{\infty} L_{1}^{*}(s, y) \mu_{2}(y) d y+\int_{0}^{\infty} L_{2}^{*}(s, x) \mu_{1}(x) d x+\varepsilon(t), \\
& L_{1}^{*}(s, 0)=\int_{0}^{\infty} \lambda_{2} r_{2} L_{0}^{*}(s, u) d u+\int_{0}^{\infty} L_{5}^{*}(s, z) \alpha(z) d z, \\
& L_{2}^{*}(s, 0)=\int_{0}^{\infty} \lambda_{1} r_{1} L_{0}^{*}(s, u) d u, \\
& L_{5}^{*}(s, 0)=\lambda_{2} r_{2} L_{3}^{*}(s), \\
& L_{6}^{*}(s, 0)=\lambda_{1} r_{1} L_{3}^{*}(s) .
\end{aligned}
$$

From previous equations, we defined the reliability function as follows: $R^{*}(s)=\int_{0}^{\infty} L_{0}^{*}(s, u) d u+\int_{0}^{\infty} L_{1}^{*}(s, y) d y+\int_{0}^{\infty} L_{2}^{*}(s, x) d x+\sum_{i=5}^{6} \int_{0}^{\infty} L_{i}^{*}(s, z) d z+L_{3}^{*}(s)$.

and the mean time to the first failure of the system (MTTFF) is given by

$$
\operatorname{MTTFF}=\lim _{s \rightarrow 0} R^{*}(s) .
$$

where,

$$
\begin{aligned}
& L_{0}^{*}(s)=\int_{0}^{\infty} L_{0}^{*}(s, u) d u=L_{0}^{*}(s, 0) \bar{V}_{1}^{*}\left(s+r_{1} \lambda_{1}+r_{2} \lambda_{2}\right), L_{1}^{*}(s)=\int_{0}^{\infty} L_{1}^{*}(s, y) d y= \\
& L_{1}^{*}(s, 0) \bar{H}_{2}^{*}\left(s+r_{1} \lambda_{1}\right), L_{2}^{*}(s)=\int_{0}^{\infty} L_{2}^{*}(s, x) d x=L_{2}^{*}(s, 0) \bar{H}_{1}^{*}\left(s+r_{2} \lambda_{2}\right), L_{3}^{*}(s)= \\
& \frac{L_{0}^{*}(s, 0) v_{1}^{*}\left(s+r_{1} \lambda_{1}+r_{2} \lambda_{2}\right)}{s+r_{1} \lambda_{1}+r_{2} \lambda_{2}}, \\
& L_{5}^{*}(s)=\int_{0}^{\infty} L_{5}^{*}(s, z) d z=L_{5}^{*}(s, 0) \bar{V}_{2}^{*}\left(s+r_{1} \lambda_{1}\right), L_{6}^{*}(s)=\int_{0}^{\infty} L_{6}^{*}(s, z) d z=L_{6}^{*}(s, 0) \bar{V}_{2}(s+ \\
& \left.r_{2} \lambda_{2}\right), \\
& L_{0}^{*}(s, 0)=\frac{-\left\{\varepsilon^{*}(s)\left(s+r_{1} \lambda_{1}+r_{2} \lambda_{2}\right)\right\}}{\left\{-s+r_{2} \lambda_{2}\left(-1+h_{2}^{*}\left(s+r_{1} \lambda_{1}\right) v_{1}^{*}\left(s+r_{1} \lambda_{1}+r_{2} \lambda_{2}\right) v_{2}^{*}\left(s+r_{1} \lambda_{1}\right)\right)\right.}+r_{1} \lambda_{1}\left(-1+h_{1}^{*}\left(s+r_{2} \lambda_{2}\right) v_{1}^{*}(s+\right. \\
& \left.\left.r_{1} \lambda_{1}+r_{2} \lambda_{2}\right) v_{2}^{*}\left(s+r_{2} \lambda_{2}\right)\right)+\left(s+r_{1} \lambda_{1}+r_{2} \lambda_{2}\right)\left(r_{1} \lambda_{1} h_{1}^{*}\left(s+r_{2} \lambda_{2}\right),\right.
\end{aligned}
$$




$$
\begin{aligned}
& \left.\left.+r_{2} \lambda_{2} h_{2}^{*}\left(s+r_{1} \lambda_{1}\right)\right) \bar{V}_{1}^{*}\left(s+r_{1} \lambda_{1}+r_{2} \lambda_{2}\right)\right\}, L_{1}^{*}(s, 0)=r_{2} \lambda_{2} L_{0}^{*}(s, 0)\left\{\bar{V}_{1}^{*}\left(s+r_{1} \lambda_{1}+r_{2} \lambda_{2}\right)+\right. \\
& \left.\left\{\frac{v_{1}^{*}\left(s+r_{1} \lambda_{1}+r_{2} \lambda_{2}\right) v_{2}^{*}\left(s+r_{2} \lambda_{2}\right)}{\left(s+r_{1} \lambda_{1}+r_{2} \lambda_{2}\right)}\right\}\right\}, \\
& L_{2}^{*}(s, 0)=r_{1} \lambda_{1} L_{0}^{*}(s, 0)\left\{\bar{V}_{1}^{*}\left(s+r_{1} \lambda_{1}+r_{2} \lambda_{2}\right)+\left\{v_{1}^{*}\left(s+r_{1} \lambda_{1}+r_{2} \lambda_{2}\right) v_{2}^{*}\left(s+r_{2} \lambda_{2}\right) /\left(s+r_{1} \lambda_{1}+r_{2} \lambda_{2}\right)\right\}\right\} \\
& L_{5}^{*}(s, 0)=\frac{\left\{r_{2} \lambda_{2} L_{0}^{*}(s, 0) v_{1}^{*}\left(s+r_{1} \lambda_{1}+r_{2} \lambda_{2}\right)\right\}}{\left(s+r_{1} \lambda_{1}+r_{2} \lambda_{2}\right)}, \\
& L_{6}^{*}(s, 0)=\frac{\left\{r_{1} \lambda_{1} L_{0}^{*}(s, 0) v_{1}^{*}\left(s+r_{1} \lambda_{1}+r_{2} \lambda_{2}\right)\right\}}{\left(s+r_{1} \lambda_{1}+r_{2} \lambda_{2}\right)} .
\end{aligned}
$$

\section{Special Case}

In this section, we present the following special cases, which confirm the results of the previous sections.

Case 1: $r_{1}=r_{2}, \phi(u)=1$, then it means any shock will cause the active units to fail and the repairman is in the system.

Case 2: $r_{1}>r_{2}, \phi(u)=1$, then it means the unit A failure is faster than unit B failure and the repairman is in the system.

Case 3: $r_{2}>r_{1}, \phi(u)=1$, then it means the unit B failure is faster than unit A failure and the repairman is in the system.

Corresponding results can easily get for the previous particular cases.

\section{Numerical Illustration}

This section shows the usefulness of the proposed system by examining the impact of the repairman and other parameters on the system through the following numerical illustrations taking into consideration that:

$V_{1}(t)=\left\{\begin{array}{cc}1-e^{-\phi} & t>0 \\ 0 & t \leq 0\end{array}, \quad V_{2}(t)=\left\{\begin{array}{cc}1-e^{-\alpha} & t>0 \\ 0 & t \leq 0^{\prime}\end{array} \quad H_{i}(t)=\left\{\begin{array}{cc}1-e^{-\mu_{i} t} & t>0 \\ 0 & t \leq 0\end{array}, \forall i=\right.\right.\right.$

\section{1,2 ,}

At first, we show Illustrative numerical examples comparing the reliability metrics for the above special cases when the repairman is present in the system. Figs (1-3) we can observe the effect of $\alpha$ when it has the values of $\{1,7,14,21,28\}$ on steady-state availability when $\lambda_{1} \in[0,1]$. From the curves of Figs (1-3), we conclude that the steady-state availability for this system increases very slowly when $\alpha>7$. Fig.4 illustrates the effect of both $r_{1}$ and $r_{2}$ on steady-state availability when $\lambda_{1} \in[0,1]$. In curve of Fig.4, we deduce that the steady-state availability is increasing when $r_{1}>r_{2}$. The idea is also clear when examining Figs. (5-7). In these Figs.(5-7) the impact of $\alpha$ when it has the values of $\{1,7,14,21,28\}$ on mean time to system failure when $\lambda_{1} \in[0,1]$. The curves of Figs.(5-7) show that the mean time to system failure is more stable when $\alpha>7$. The curve of Fig.8 shows that the mean time to system failure increases when $r_{1}<r_{2}$ in interval $\lambda_{1} \in[0,0.35]$ as it is increases also when $r_{1}>r_{2}$ in interval $\lambda_{1} \in[0.35,1]$.

The steady-state availability and the mean time to system failure are examined, when $\lambda_{1}$ and $\alpha$ change, as shown in Tables (1-6). We vary the values of $\lambda_{1}$ 
and $\alpha$ and note their cross-impact on the steady-state availability and the mean time to system failure. It shows that increasing $\lambda_{1}$ can greatly decrease the steady-state availability and the mean time to system failure; however, increasing $\alpha$ seldom affects the values of steady-state availability and the mean time to system failure.

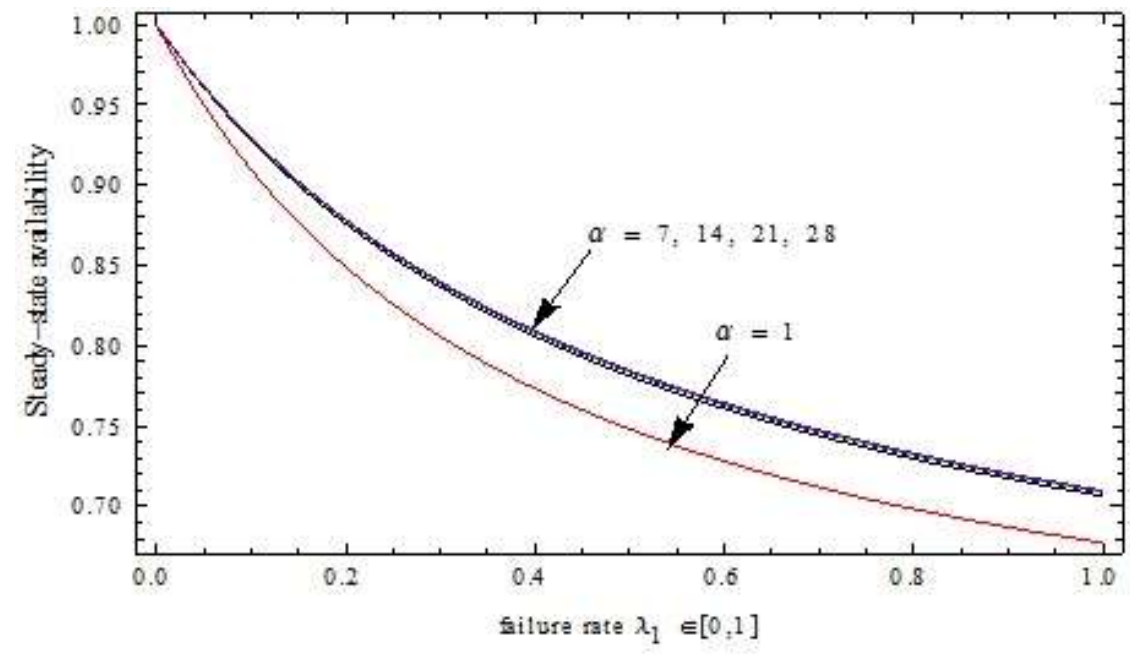

Fig. 1. Steady-state availability versus rate $\lambda_{1}$ when $r_{1}=r_{2}$ and parameter $\alpha=1,7,14,21,28$

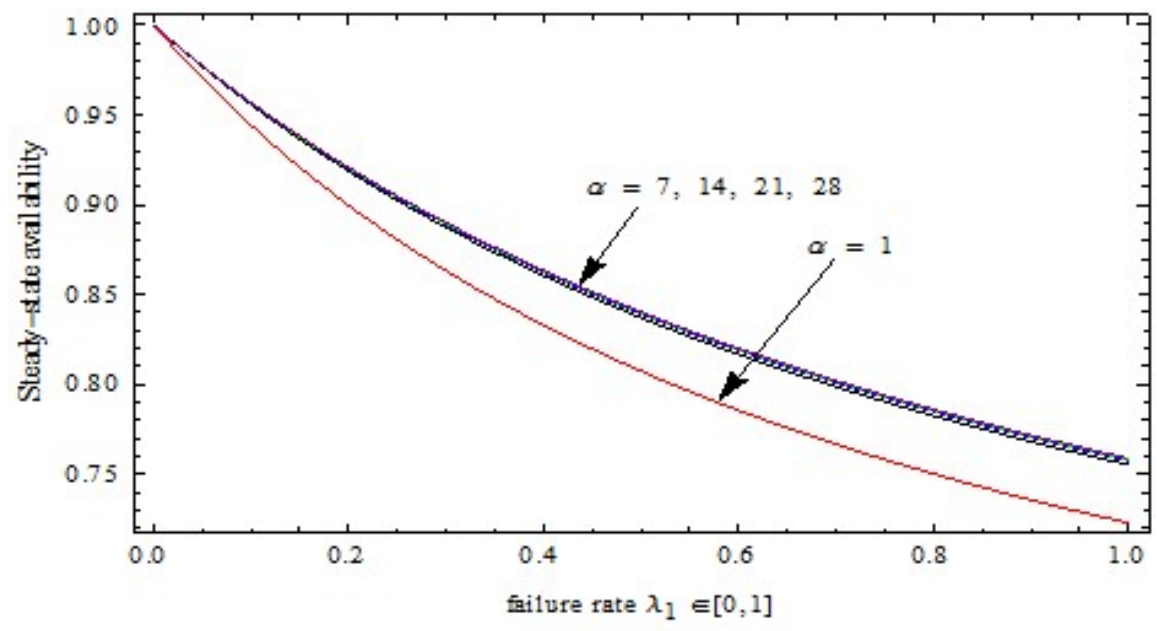

Fig. 2. Steady-state availability versus rate $\lambda_{1}$ when $r_{1}<r_{2}$ and parameter $\alpha=1,7,14,21,28$ 


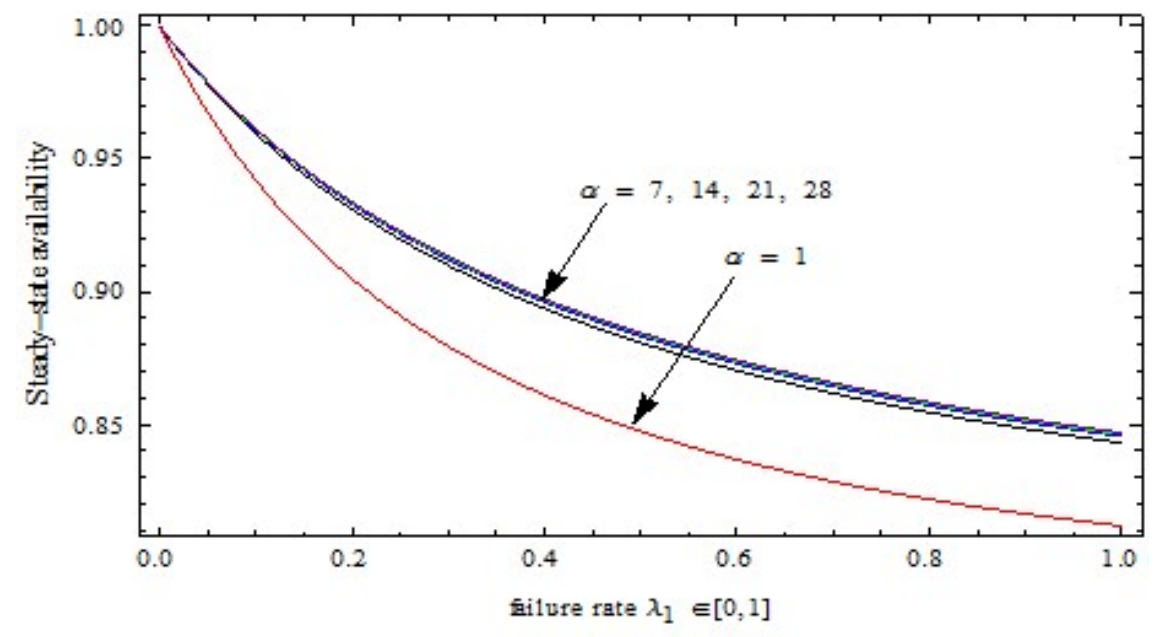

Fig 3. Steady-state availability versus rate $\lambda_{1}$ when $r_{1}>r_{2}$ and parameter $\alpha=1,7,14,21,28$

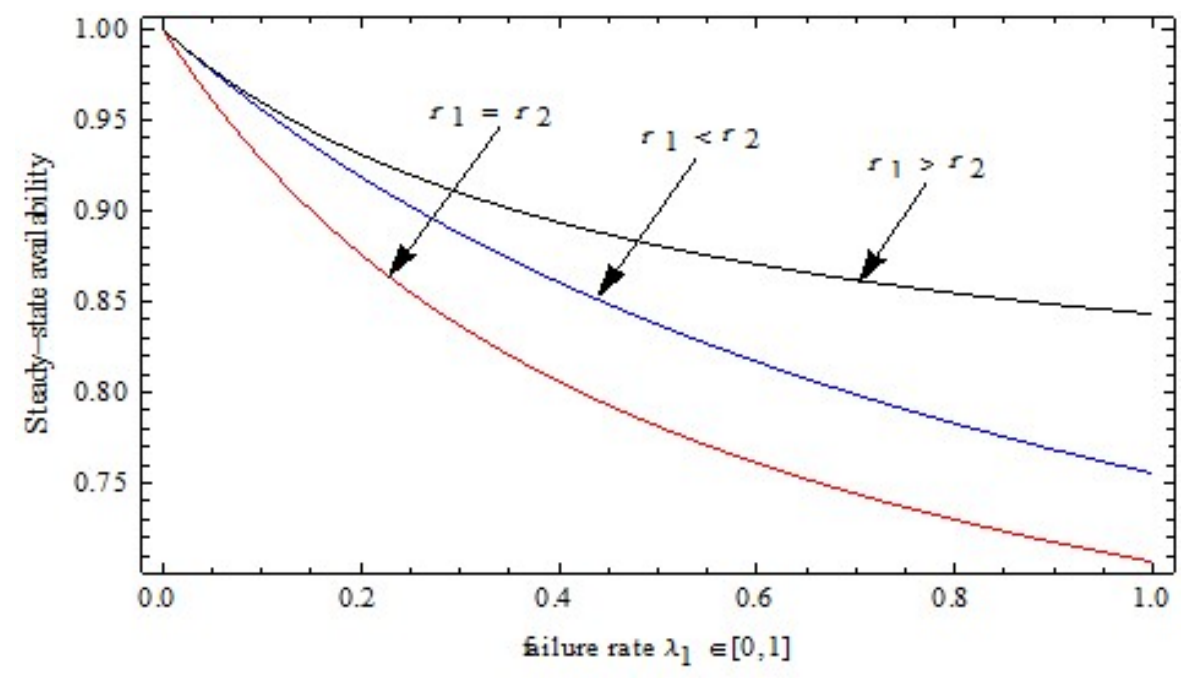

Fig 4. Steady-state availability versus rate $\lambda_{1}$ when $\alpha=7$

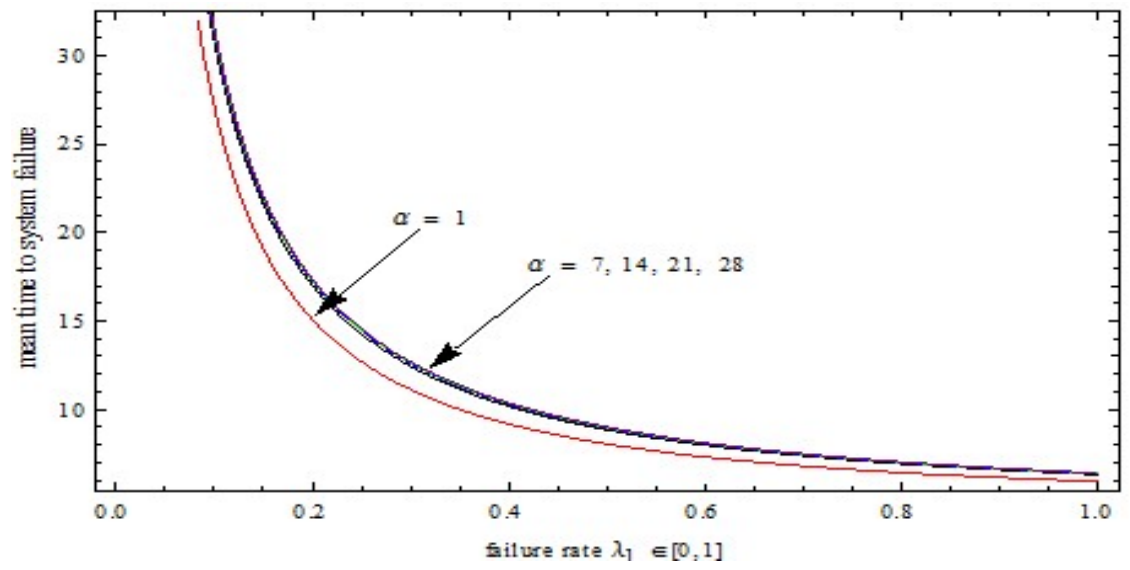

Fig. 5. Mean time to the first failure versus rate $\lambda_{1}$ when $r_{1}=r_{2}$ and parameter $\alpha=1,7,14,21,28$ 


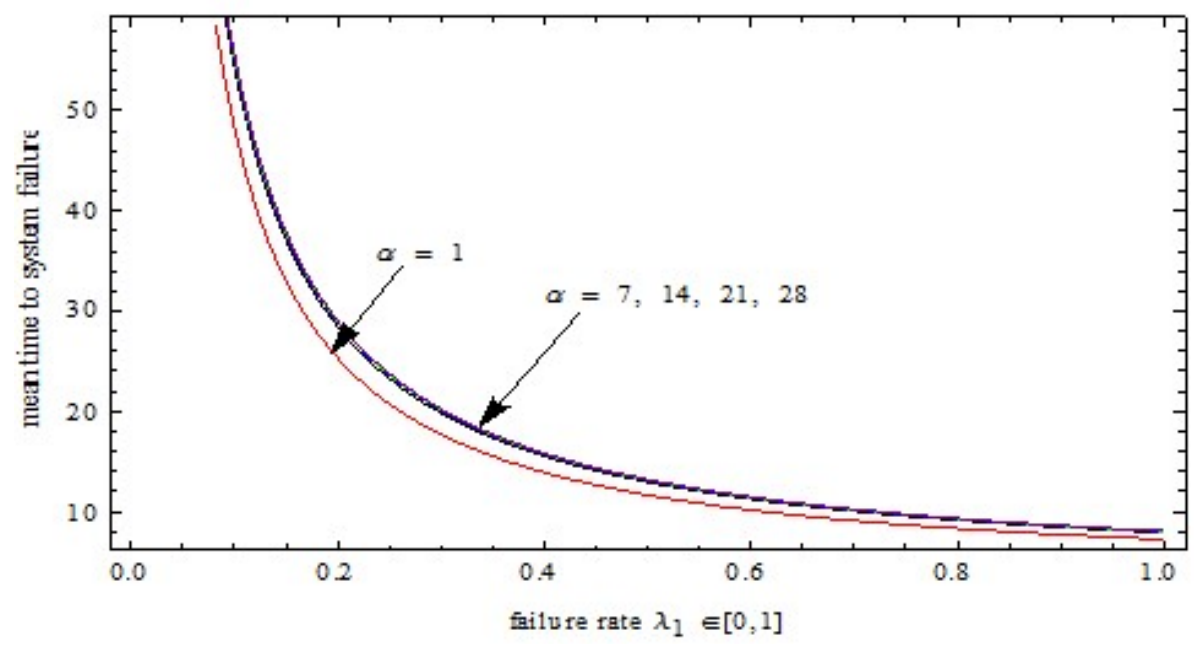

Fig. 6. Mean time to the first failure versus rate $\lambda_{1}$ when $r_{1}<r_{2}$ and parameter $\alpha=1,7,14,21,28$

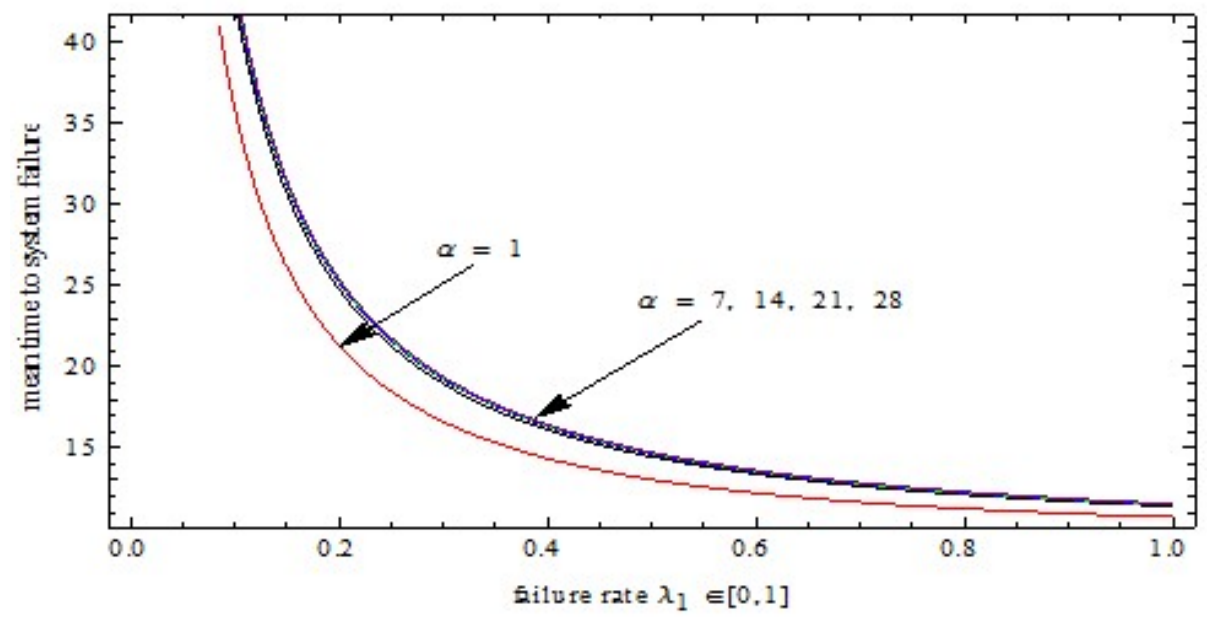

Fig. 7. Mean time to the first failure versus rate $\lambda_{1}$ when $r_{1}>r_{2}$ and parameter $\alpha=1,7,14,21,28$.

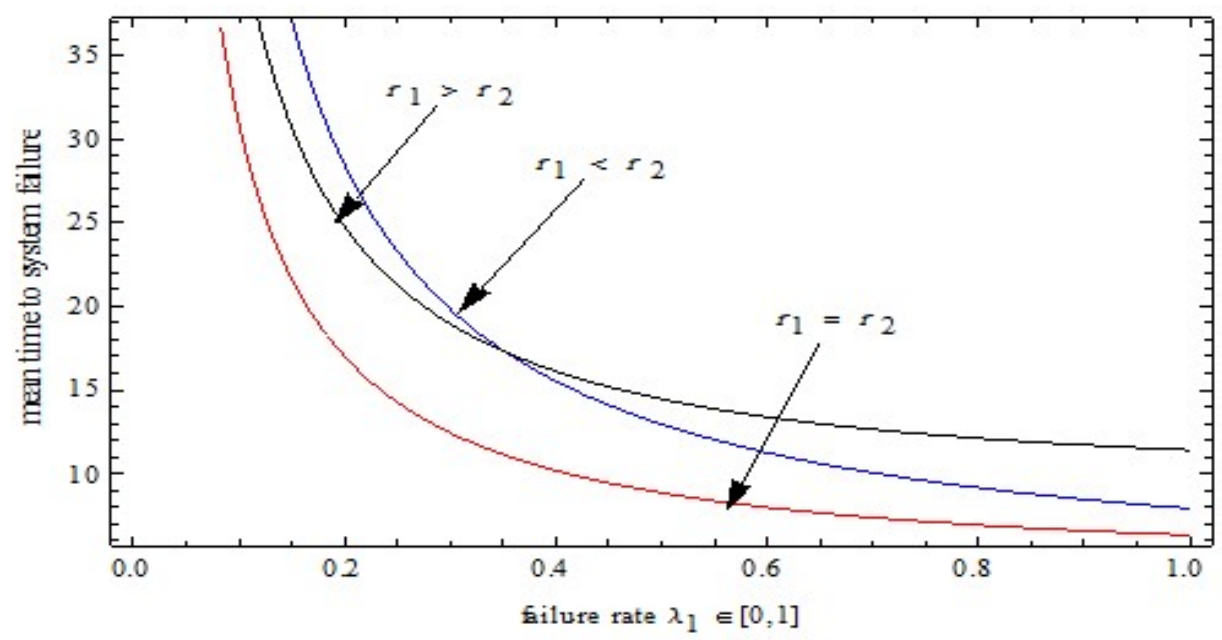

Fig 8. Mean time to the first failure versus rate $\lambda_{1}$ when $\alpha=7$ 
Table 1. Steady-state availability for different a and $r_{1}=0.3<r_{2}=0.7$ when $\lambda_{2}=0.4, p=0.3, q=0.7, \mu_{2}=0.4, \mu_{1}=0.5$

\begin{tabular}{ccccccccc}
\hline$\lambda_{1}$ & $\alpha=1$ & $\alpha=7$ & $\alpha=14$ & $\alpha=21$ & $\alpha=28$ & $\alpha=35$ & $\alpha=42$ & $\alpha=49$ \\
0.1 & 0.945163 & 0.955857 & 0.956584 & 0.956819 & 0.956935 & 0.957004 & 0.95705 & 0.957083 \\
0.2 & 0.900995 & 0.918948 & 0.92018 & 0.920579 & 0.920776 & 0.920893 & 0.920971 & 0.921026 \\
0.3 & 0.864701 & 0.887634 & 0.889219 & 0.889732 & 0.889986 & 0.890137 & 0.890237 & 0.890309 \\
0.4 & 0.834379 & 0.860735 & 0.862568 & 0.863161 & 0.863454 & 0.863629 & 0.863745 & 0.863827 \\
0.5 & 0.808691 & 0.83738 & 0.839385 & 0.840034 & 0.840355 & 0.840546 & 0.840673 & 0.840763 \\
0.6 & 0.786666 & 0.816913 & 0.819037 & 0.819724 & 0.820064 & 0.820266 & 0.820401 & 0.820497 \\
0.7 & 0.767587 & 0.798832 & 0.801034 & 0.801747 & 0.802099 & 0.802309 & 0.802449 & 0.802548 \\
0.8 & 0.75091 & 0.782743 & 0.784994 & 0.785723 & 0.786083 & 0.786297 & 0.78644 & 0.786541 \\
\hline
\end{tabular}

Table 2. Steady-state availability for different a and $r_{1}=0.7>r_{2}=0.3$ when $\lambda_{2}=0.4, p=0.7, q=0.3, \mu_{2}=0.4, \mu_{1}=0.5$

\begin{tabular}{ccccccccc}
\hline$\lambda_{1}$ & $\alpha=1$ & $\alpha=7$ & $\alpha=14$ & $\alpha=21$ & $\alpha=28$ & $\alpha=35$ & $\alpha=42$ & $\alpha=49$ \\
0.1 & 0.94198 & 0.959523 & 0.960715 & 0.961101 & 0.961292 & 0.961405 & 0.961481 & 0.961535 \\
0.2 & 0.904875 & 0.931008 & 0.932854 & 0.933453 & 0.93375 & 0.933927 & 0.934045 & 0.934129 \\
0.3 & 0.879432 & 0.909885 & 0.912099 & 0.912822 & 0.91318 & 0.913393 & 0.913535 & 0.913637 \\
0.4 & 0.861099 & 0.893637 & 0.89606 & 0.896853 & 0.897246 & 0.897481 & 0.897638 & 0.897749 \\
0.5 & 0.847388 & 0.88077 & 0.883306 & 0.884137 & 0.88455 & 0.884797 & 0.884962 & 0.885079 \\
0.6 & 0.836832 & 0.87034 & 0.872928 & 0.873779 & 0.874202 & 0.874455 & 0.874623 & 0.874743 \\
0.7 & 0.82851 & 0.861724 & 0.864325 & 0.865182 & 0.865608 & 0.865864 & 0.866034 & 0.866155 \\
0.8 & 0.821822 & 0.854491 & 0.85708 & 0.857935 & 0.858361 & 0.858617 & 0.858786 & 0.858908 \\
\hline
\end{tabular}

Table 3. Steady-state availability for different a and $r_{1}=r_{2}=0.6$ when $\lambda_{2}=0.4, p=0.5, q=0.5, \mu_{2}=0.4, \mu_{1}=0.5$

\begin{tabular}{ccccccccc}
\hline$\lambda_{1}$ & $\alpha=1$ & $\alpha=7$ & $\alpha=14$ & $\alpha=21$ & $\alpha=28$ & $\alpha=35$ & $\alpha=42$ & $\alpha=49$ \\
0.1 & 0.909832 & 0.927946 & 0.929189 & 0.929592 & 0.92979 & 0.929909 & 0.929988 & 0.930044 \\
0.2 & 0.849187 & 0.875919 & 0.877782 & 0.878386 & 0.878684 & 0.878862 & 0.87898 & 0.879064 \\
0.3 & 0.805794 & 0.836605 & 0.838777 & 0.839481 & 0.839829 & 0.840037 & 0.840174 & 0.840272 \\
0.4 & 0.773317 & 0.805862 & 0.808177 & 0.808927 & 0.809298 & 0.809519 & 0.809666 & 0.809771 \\
0.5 & 0.748162 & 0.781169 & 0.783533 & 0.7843 & 0.784679 & 0.784904 & 0.785055 & 0.785161 \\
0.6 & 0.728147 & 0.760904 & 0.763264 & 0.764029 & 0.764408 & 0.764634 & 0.764784 & 0.76489 \\
0.7 & 0.711866 & 0.743977 & 0.746302 & 0.747056 & 0.747429 & 0.747652 & 0.747799 & 0.747905 \\
0.8 & 0.698382 & 0.729627 & 0.731899 & 0.732637 & 0.733002 & 0.733219 & 0.733364 & 0.733467 \\
\hline
\end{tabular}

Table 4. Mean time to the first failure for different a and $r_{1}=0.3<r_{2}=0.7$ when $\lambda_{2}=0.4, p=0.5, q=0.5, \mu_{2}=0.4, \mu_{1}=$

\begin{tabular}{ccccccccc}
\hline \multicolumn{1}{c}{0.5} \\
\hline$\lambda_{1}$ & $\alpha=1$ & $\alpha=7$ & $\alpha=14$ & $\alpha=21$ & $\alpha=28$ & $\alpha=35$ & $\alpha=42$ & $\alpha=49$ \\
0.1 & 48.3086 & 54.3999 & 55.1436 & 55.4034 & 55.5357 & 55.6158 & 55.6696 & 55.7081 \\
0.2 & 25.3126 & 28.4357 & 28.8173 & 28.9506 & 29.0184 & 29.0596 & 29.0871 & 29.1069 \\
0.3 & 17.6815 & 19.8073 & 20.0674 & 20.1584 & 20.2047 & 20.2327 & 20.2515 & 20.265 \\
0.4 & 13.8895 & 15.5115 & 15.7104 & 15.78 & 15.8154 & 15.8369 & 15.8513 & 15.8616 \\
0.5 & 11.6318 & 12.9476 & 13.1095 & 13.1662 & 13.195 & 13.2125 & 13.2242 & 13.2327 \\
0.6 & 10.14 & 11.249 & 11.386 & 11.4339 & 11.4583 & 11.4732 & 11.4831 & 11.4902 \\
\hline
\end{tabular}




\begin{tabular}{lllllllll}
\hline 0.7 & 9.08501 & 10.0442 & 10.1631 & 10.2048 & 10.2261 & 10.2389 & 10.2476 & 10.2538 \\
0.8 & 8.30235 & 9.1475 & 9.25282 & 9.28974 & 9.30855 & 9.31996 & 9.32762 & 9.33311 \\
\hline
\end{tabular}

Table 5. Mean time to the first failure for different a and $r_{1}=0.7>r_{2}=0.3$ when $\lambda_{2}=0.4, p=0.7, q=0.3, \mu_{2}=0.4, \mu_{1}=$

\begin{tabular}{ccccccccc}
\multicolumn{10}{c}{0.5} \\
\hline$\lambda_{1}$ & $\alpha=1$ & $\alpha=7$ & $\alpha=14$ & $\alpha=21$ & $\alpha=28$ & $\alpha=35$ & $\alpha=42$ & $\alpha=49$ \\
0.1 & 35.8646 & 42.5894 & 43.3861 & 43.6633 & 43.8042 & 43.8894 & 43.9466 & 43.9876 \\
0.2 & 21.3104 & 24.7677 & 25.181 & 25.3249 & 25.3982 & 25.4425 & 25.4722 & 25.4935 \\
0.3 & 16.6047 & 18.9434 & 19.2262 & 19.3249 & 19.3752 & 19.4056 & 19.426 & 19.4406 \\
0.4 & 14.3401 & 16.104 & 16.3201 & 16.3957 & 16.4342 & 16.4575 & 16.4732 & 16.4844 \\
0.5 & 13.0387 & 14.4495 & 14.6247 & 14.6861 & 14.7174 & 14.7363 & 14.7491 & 14.7582 \\
0.6 & 12.2105 & 13.3813 & 13.5286 & 13.5804 & 13.6068 & 13.6228 & 13.6335 & 13.6412 \\
0.7 & 11.6469 & 12.6439 & 12.771 & 12.8157 & 12.8385 & 12.8524 & 12.8617 & 12.8684 \\
0.8 & 11.2448 & 12.1102 & 12.2218 & 12.2612 & 12.2813 & 12.2935 & 12.3017 & 12.3076 \\
\hline
\end{tabular}

Table 6. Mean time to the first failure for different a and $r_{1}=r_{2}=0.6$ when $\lambda_{2}=0.4, p=0.5, q=0.5, \mu_{2}=0.4, \mu_{1}=0.5$

\begin{tabular}{lcccccccc}
\hline$\lambda_{1}$ & $\alpha=1$ & $\alpha=7$ & $\alpha=14$ & $\alpha=21$ & $\alpha=28$ & $\alpha=35$ & $\alpha=42$ & $\alpha=49$ \\
0.1 & 27.171 & 30.8908 & 31.341 & 31.4981 & 31.5781 & 31.6265 & 31.659 & 31.6822 \\
0.2 & 15.074 & 17.0007 & 17.235 & 17.3169 & 17.3585 & 17.3837 & 17.4007 & 17.4128 \\
0.3 & 11.1095 & 12.4238 & 12.5849 & 12.6412 & 12.6698 & 12.6872 & 12.6989 & 12.7072 \\
0.4 & 9.17023 & 10.1699 & 10.2935 & 10.3368 & 10.3589 & 10.3722 & 10.3812 & 10.3876 \\
0.5 & 8.03594 & 8.84161 & 8.9423 & 8.97761 & 8.99561 & 9.00652 & 9.01384 & 9.0191 \\
0.6 & 7.30062 & 7.97369 & 8.05874 & 8.08862 & 8.10386 & 8.1131 & 8.11931 & 8.12376 \\
0.7 & 6.79076 & 7.36705 & 7.4407 & 7.46662 & 7.47985 & 7.48788 & 7.49327 & 7.49714 \\
0.8 & 6.42001 & 6.9224 & 6.98732 & 7.01021 & 7.02191 & 7.02901 & 7.03378 & 7.0372 \\
\hline
\end{tabular}

\section{Conclusion}

In this article, we deduced the reliability measurements of a system consisting of two dissimilar parallel units and a single repairman. The repairman might take a vacation or not at the beginning of the system operation and the active units might be attacked from successive shocks. Such a system can be considered as an evolution of a general repairable Industrial system and is also difficult to theoretically analyze the existence of many random variables with general distributions. The numerical illustration explains the relationship between the derived reliability measurements and system parameters.

\section{Acknowledgments}

This research was funded by the Deanship of Scientific Research (DSR) at King Abdulaziz University, Jeddah, under Grant No. G:131-662-1439. The author, therefore, acknowledges with thanks DSR for technical and financial support.

\section{References}

1. Yonatan Levy \& Uri Yechiali. Utilization of the idle time in an M/G/1 queue, Management Science. Management Science. 1975, Volume 22, pp. 202-211.

2. Tian Naishuo \& Zhang George. Vacation Queueing Models-Theory and Applications. January 2006, Springer-Verlag, New York. 
3. Su, B. H., Shi, D. H. Reliability analysis of n-unit series systems with multiple vacations of a repairman. Mathematical Statistics and Applied Probability. 1995, Volume 10, pp. 78-82.

4. Jau-Chuan Ke, Chuen-Horng Lin. A Markov repairable system involving an imperfect service station with multiple vacations. Asia Pacific Journal of Operational Research. 2005, Volume 22, pp. 555-582.

5. Hu, L. M., Li, J. D. Reliability analysis of a three-unit system with vacation and priority. ICIC Express Letters. 2009, Volume 3, pp. 171-176.

6. Linmin $\mathrm{Hu}$, Jiandong $\mathrm{Li}$, Wenming Fang. Reliability analysis of an n-component series system with $\mathrm{m}$ failure modes and vacation. ICIC Express Letters. 2008, Volume 2, pp. 53-58.

7. Liu, R. B., Tang,Y. H., Luo, C. Y. A new kind of n-unit series repairable system and its reliability analysis. Mathematica Applicata. 2007, Volume 20, pp. 164-170.

8. Renbin Liu. \& Zaiming Liu. Probability Analysis of a Two-unit Basic Model with a Repairman Who Takes Multiple Vacations. Journal of Information \& Computational Science. 2011, Volume 8, pp. 554-561.

9. Yan Ling Lia \& Gen Qi Xu. Analysis of two components parallel repairable system with vacation. Communications in Statistics -Theory and Methods. 27 Sep 2019, DOI:10.1080/03610926.2019.1670847.

10. Nakagawa \& Osaki S., Stochastic behavior of two-unit paralleled redundant system with repair maintenance. Microelectronics and Reliability. 1975, Volume 14, pp. 457-461.

11. Shi, D.-H. Analysis of a two-unit series repairable model. Acta Automatica Sinica. 1985, Volume 11, pp. 71-79.

12. Ke, J.C., Wang, K.H. Vacation policies for machine repair problem with two type spares. Applied Mathematical Modelling. 2007, Volume 31, pp. 880-894.

13. Mohamed Salah El-Sherbeny. Stochastic Behavior of a Two-Unit Cold Standby Redundant System Under Poisson Shocks. Arabian Journal for Science and Engineering. 2017, Volume 42, pp.3043-3053.

14. Yutian Chen, Xianyun Meng \& Shengqiang Chen. Reliability Analysis of a Cold Standby System with Imperfect Repair and under Poisson Shocks. Mathematical Problems in Engineering. 2014, Volume 3, pp.1-11.

15. Xin Ge., Jiali Sun., \& Qingtai Wu. Reliability analysis for a cold standby system under stepwise Poisson shocks. Journal of control and decision. 23 Jun 2019, DOI:10.1080/23307706.2019.1633961.

16. Xiaolin Liang., Lanying Mo., \& Xiaowei Tang. Study on a cold standby repairable deteriorating system with repairman vacation. journal of system engineering. 2010, Volume 25, pp. 426-432.

17. Mahmoud, M.A.W., Rashad, A.M., Zienab Hussien. Stochastic Analysis of a Repairable Cold Standby System Attacked by Poisson Shocks Considering Inspection and Post Repair. International Journal of Computer Applications. 2015, Volume 132, pp.33-40. 